\title{
THE PLASMA VISCOSITY
}

\author{
BY \\ J. S. LAWRENCE \\ From the Rheumatism Research Centre, Manchester University
}

(RECEIVED FOR PUBLICATION JUNE 15, 1950)

The viscosity of the plasma was studied briefly by Fahraeus in his original investigations (1921) into the blood sedimentation rate. In 1940 further studies were made by T'Ang and Wang, who concluded that it gave a more accurate picture of the activity of disease processes than the erythrocyte sedimentation rate. The plasma viscosity technique was independently developed by Whittington and his colleagues, who reached a similar conclusion (Miller and Whittington, 1942 ; Houston, Harkness, and Whittington, 1946).

In the present work an attempt has been made to study the importance of individual protein fractions in plasma viscosity changes and to assess the value of such a fractionation in diagnosis, prognosis, and the control of therapy.

\section{Material}

In all, viscosity measurements have been made in some 700 samples of plasma, and the complete fractional viscosity method has been studied in 292 samples, 47 from healthy subjects and the remainder from a wide variety of diseases. These include the following:

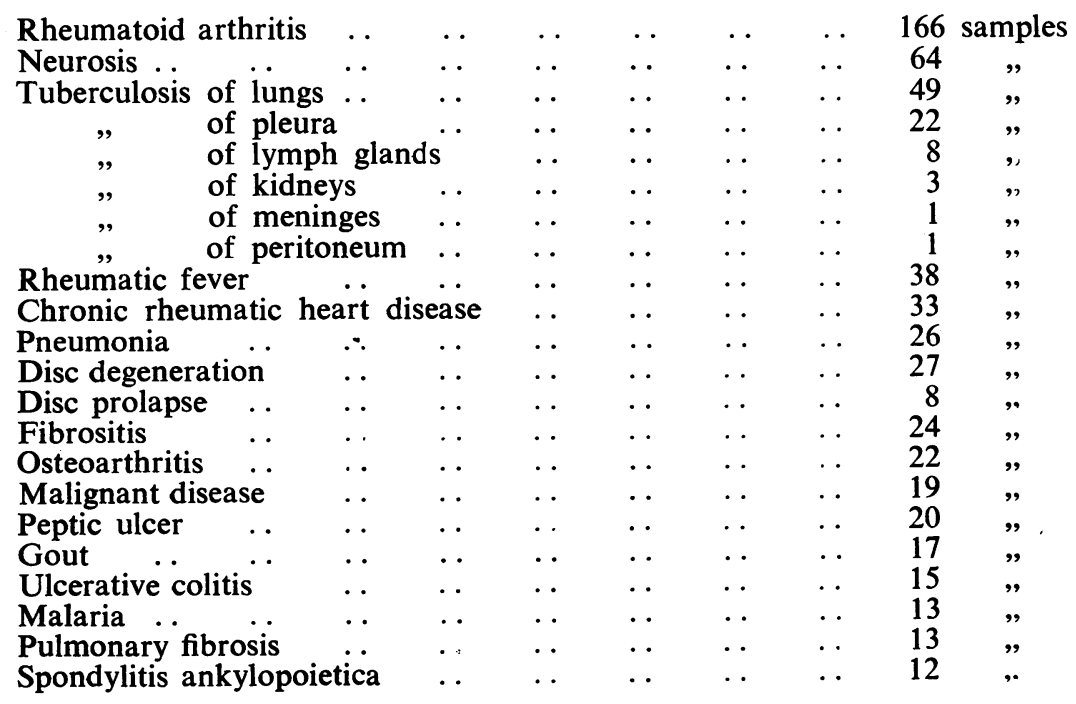




\begin{tabular}{|c|c|c|c|c|c|c|c|c|}
\hline Abscesses and carbun & les & & & $\cdot$ & & & 11 & samples \\
\hline Hepatitis (acute and & sub & & & . & . & $\ldots$ & 9 & ,, \\
\hline Bronchitis $\quad \ldots$ & .. & .. & . & . & .. & .. & 9 & ," \\
\hline Bronchiectasis & .. & $\cdots$ & . & $\cdots$ & $\because$ & $\cdots$ & 7 & $"$ \\
\hline Traumatic disorders & .. & $\cdots$ & $\cdot$ & . & .. & .. & 7 & , \\
\hline Tertiary syphilis & . & $\cdots$ & . & . & . & .. & 6 & 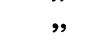 \\
\hline Asthma & . & . & . & . & . & . & 5 & ” \\
\hline Hypertension & . & . & . & .. & .. & . & 6 & $"$ \\
\hline Coronary thrombosis & $\ldots$ & $\ldots$ & . & .. & . & . & 5 & ", \\
\hline Nephritis & . & . & . & 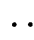 & .. & $\cdots$ & 4 & " \\
\hline Urticaria and angio- $n$ & uro & oed & & $\cdots$ & . & . & 4 & , \\
\hline Amoebiasis & & . & & . & 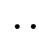 & & 4 & , \\
\hline Miscellaneous disorder & & & . & & & & 108 & , \\
\hline
\end{tabular}

\section{Method}

Fractionation.-Using minimal stasis, $10 \mathrm{ml}$. of blood was collected in a syringe previously rinsed with $3.8 \%$ sodium citrate. The blood was placed in a tube containing $0.1 \mathrm{ml}$. of double strength oxalate mixture, ${ }^{*}$ well mixed, and then centrifuged to separate the plasma. The viscosity of the oxalate plasma was estimated. Calcium chloride (in this instance $0.03 \mathrm{ml}$. of $40 \%$ calcium chloride) was then added to precipitate the oxalate and induce clotting. (An excess must be avoided, as it maintains the fibrin in solution.) Half an hour is required for clotting at $37^{\circ} \mathrm{C}$., or the sample may be left overnight at room temperature. The serum was obtained by expressing the clot and its viscosity estimated. A $4 M$-ammonium sulphate solution was then added in a proportion of one volume of this to two volumes of plasma. In this way a portion of the serum globulin was precipitated. This is identical with the fraction separated by Jager and Nickerson (1948) in their method for estimating the $\gamma$ globulin and shown to consist almost entirely of this protein. The protein so precipitated was then removed by filtration, using a Whatman 40 or 50 paper, and the viscosity of the filtrate estimated. A further portion of the globulin was then removed by the addition of one volume of $4 \mathrm{M}$-ammonium sulphate to three volumes of the filtrate, thus giving a 2 molar solution-i.e., approximately half-saturated. After filtration through a No. 50 Whatman paper the viscosity of this second filtrate was then estimated. If any of the precipitate came through in the early stages of filtration the liquor was returned to the filter.

Measurement of Viscosity.-Only the capillary type of viscometer is suitable for use with human plasma. This consists essentially of two reservoirs at different heights connected by a capillary tube. In a viscometer for clinical use there are three points that require consideration: (1) The instrument should use a reasonable amount of plasma. This must be a compromise, as a large capacity is associated with smaller errors and is easier to use but is less practicable for routine clinical use on account of its demands in plasma. It is above all important that the instrument be so designed that there is no wastage of plasma. (2) The accuracy should not be greatly affected by small errors of filling so that the instrument must be washed and dried between each of a series of tests. (3) It should be capable of being immersed in water so that it may be maintained at a constant temperature.

The Hess viscometer, which was designed for use with whole blood, cannot be inserted into a water bath and so lacks the precision which is essential for use with plasma.

The Ostwald viscometer is suitable, but as generally made is wasteful of plasma and thus as a rule requires too large an amount. The Ostwald viscometer used in the

* Ammonium oxalate $6 \mathrm{~g}$. Potassium oxalate $4 \mathrm{~g}$; water to $100 \mathrm{ml}$. kept at $37^{\circ} \mathrm{C}$. Tubes should not be dried in a hot oven. 


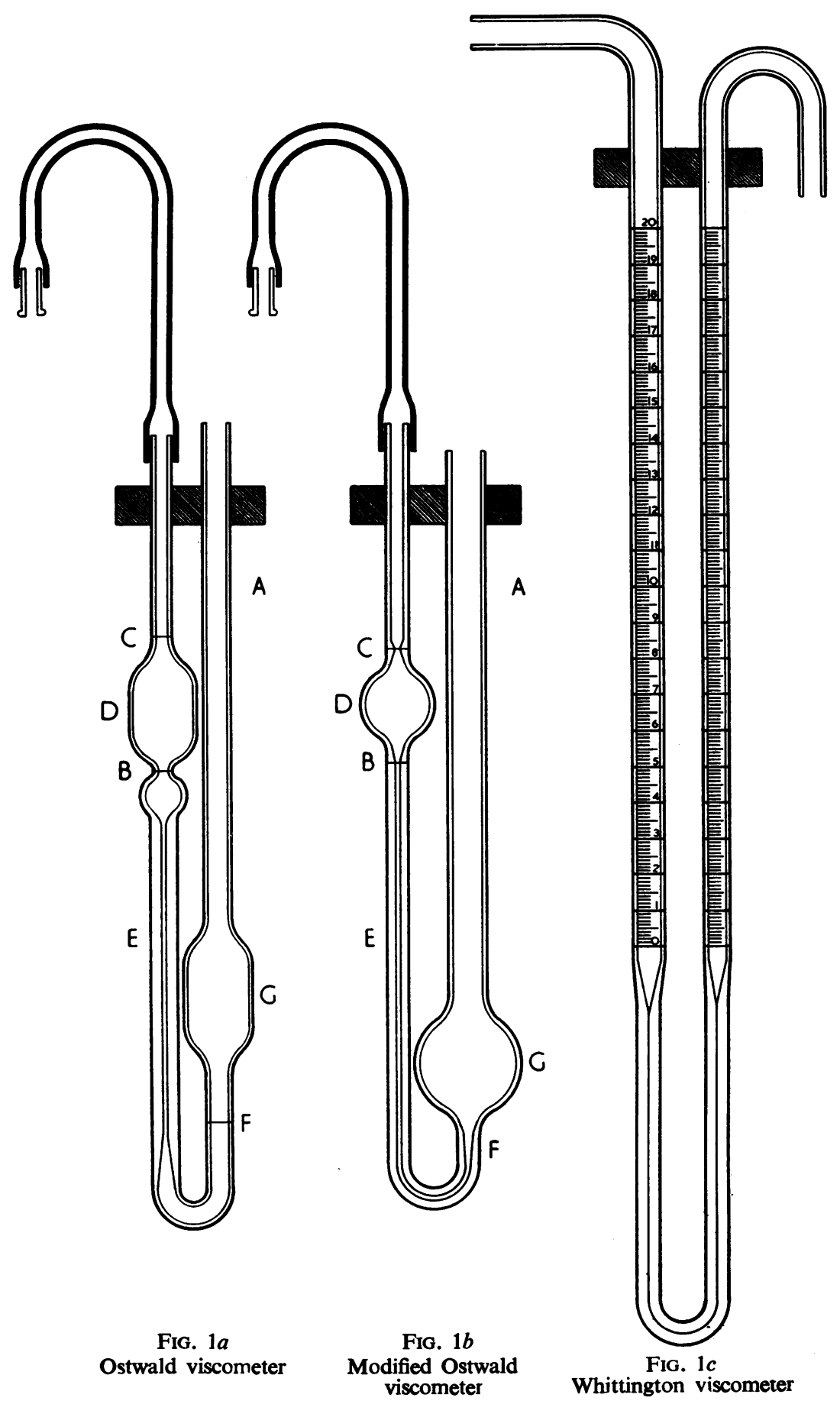

FIG. $1 a$

Ostwald viscometer 
earlier experiments is shown in Fig. $1 a^{*}$ It requires at least $6.5 \mathrm{ml}$. of plasma, so that $20 \mathrm{ml}$. of blood is required for the test, but it is simple to use and accurate. It will be observed that an appreciable amount of plasma is required to fill the tube at the U-bend between the lower end of the capillary and the mark F; this is not used in the viscosity test and is thus wasted.

A smaller instrument made to the author's design is shown in Fig. $1 b . \dagger$ It requires $2.5 \mathrm{ml}$, but this volume of plasma is almost entirely used for the test, as the capillary tubing is continuous from one bulb to the other round the U-bend. Slight errors of filling are neutralized by the wide diameter of the lower bulb, which avoids an appreciable alteration in the head of pressure from over- or under-filling. Failure to dry the instrument before filling, for example, results in an error of only $0.2 \%$. Thus, provided samples of similar viscosity are being tested, it is not necessary to wash and dry the instrument between tests. It should, however, always be washed after a high viscosity sample has been tested. Serum should never be placed in the instrument after plasma without washing, as clotting will occur. Similarly, samples containing ammonium sulphate should not be put into the instrument without washing if it has just contained serum or plasma, or serum containing a lesser saturation of ammonium sulphate, as precipitation occurs on the wall of the capillary and invalidates the result.

The Whittington viscometer (Fig. 1c) requires only $0.7 \mathrm{ml}$. of plasma, but the capillary is very fine, so that the instrument is difficult to wash and error may result from small particles of suspended matter. Owing to the small diameter of the right bend, or receiving limb, small errors of filling affect the head of pressure appreciably and must be corrected. The instrument must be washed and dried between each sample, so that much time is lost.

Temperature.-The temperature used in viscometry has unfortunately not been standardized. Most workers have used a temperature of $25^{\circ} \mathrm{C}$. as in the experiments here set forth. Whittington and his colleagues have adopted a $20^{\circ} \mathrm{C}$. standard. This is more readily maintained but less convenient in hot climates or summer weather, unless special equipment is available for cooling. It has, however, a theoretical advantage, as the viscosity of water is approximately 1 centipoise at $20^{\circ} \mathrm{C}$. so that the relative viscosity is numerically identical with the value in centipoises. This, however, is not of great importance, as, owing to the effect of varying instrument design on the kinematic viscosity of protein solutions, results cannot at present be expressed in finite units. An approximate value for the timing at $20^{\circ} \mathrm{C}$. may be obtained by adding $12.5 \%$ to the time required at $25^{\circ} \mathrm{C}$. It is of course essential to mention the temperature used when expressing results, and it would be advisable that a standard temperature be adopted by all workers.

Calibration of the Viscometer.-If results are to be comparable with those of other workers the viscometer must be calibrated against two liquids of different viscosity. One of these may be water, but the other must be a protein solution similar to plasma. Calibration with crystalloid solutions or pure liquids is of no value if a viscometer is to be used for testing protein solutions (Harkness and Whittington, 1947). Table I gives the viscosity of solutions of pure human albumin, using the author's instrument $\left(0_{1}\right)$. The viscosity is here calculated according to the formula:

$$
\begin{aligned}
& V=\frac{T_{p}}{T_{w}} \text { where } V=\text { the viscosity } \\
& T_{p}=\text { the time taken with the albumin solution } \\
& T_{w}=\text { the time taken with water }
\end{aligned}
$$

* Supplied by Messrs. Gallenkamp, London.

† Supplied by the Manchester Scientific Glassware Co., 12, Wright Street, Manchester 15. 
TABLE I

Viscosity of Pure Human Albumin (Lederle) with Viscometer $\mathbf{0}_{1}$

\begin{tabular}{c|c}
\hline Albumin (\%) & Viscosity \\
\hline 25 & 6.01 \\
20 & 3.35 \\
15 & 2.12 \\
10 & 1.54 \\
6 & 1.29 \\
5 & 1.24 \\
4 & 1.18 \\
3 & 1.13 \\
2 & 1.08 \\
0 & 1.00 \\
\hline
\end{tabular}

$($ Water $=100)$

To calibrate another instrument against viscometer $0_{1}$ the time required for water and, for example, a $10 \%$ albumin solution is determined. The value for two constants $A$ and $B$ is then calculated according to the formula $V=A T+\frac{B}{T}$. For example, if the time taken for water is 34.6 seconds and for $10 \%$ albumin 56.0 seconds with the viscometer to be calibrated, two equations are obtained.

$$
\begin{aligned}
1.00 & =\mathrm{A} \times 34.6+\frac{\mathrm{B}}{34.6} \\
\text { and } 1.54 & =\mathrm{A} \times 56.0+\frac{\mathrm{B}}{56.0}
\end{aligned}
$$

If these two equations are solved the result obtained is

$$
\text { and } \begin{aligned}
\mathrm{A} & =0.0263 \\
\mathrm{~B} & =2.8
\end{aligned}
$$

Substituting these in the formula $V=A T+\frac{B}{T}$ we have $V=0.0263 \times T+\frac{2.8}{T}$ from which the viscosity of any sample of plasma or serum may be calculated by substituting for $T$ the time taken with the unknown sample. It should be clearly understood that this gives the results in terms of the viscometer $0_{1}$ and that these need not correspond with the figures obtained if a different viscometer is used as a standard. The sole advantage of standardizing to $0_{1}$ is that the figures for normal values of the fractional viscosity given in this paper may then be used.

As an alternative to the above calculation a calibration chart may be prepared by plotting viscosity against time using the same formula.

\section{Method of Using the Ostwald Viscometer}

The viscometer is suspended in a tank holding preferably one to four gallons of water thermostatically controlled to within $\pm 0.3^{\circ} \mathrm{C}$. To carry out the tests an exact amount of plasma appropriate to the instrument-i.e., the amount used when calibrating it-is introduced into the limb A (Fig. $1 b$ ) by means of a fine-pointed pipette. If the plasma is run in carefully down the wall of the viscometer limb the formation of bubbles is avoided. After allowing three minutes for the temperature to become stabilized the plasma is sucked up to mark C. It now fills the bulb $D$ and the capillary tube $E$. A small amount remains in the lower bulb G. The fluid is released and is then timed 
with a stop watch till the mark B is reached. This time, which should be accurate to 0.1 seconds, is then substituted for $\mathrm{T}$ in the formula $\mathrm{V}=\mathrm{AT}+\frac{\mathrm{B}}{\mathrm{T}}$, or a calibration chart as already described may be used. On completion of the test the viscometer is dried on the outside and emptied by blowing gently through the mouthpiece. It is washed out first with $2.5 \%$ sodium hydroxide, then with water.

\section{Calculation}

As already described, figures were obtained for the plasma, serum, and two filtrates, and the viscosity differences due to fibrinogen, two fractions of globulin, and the albumin can then be estimated. The following example illustrates the method of calculating the relative viscosity:

\begin{tabular}{c|c|c|c}
\hline Plasma & Serum & First Filtrate & Second Filtrate \\
\cline { 2 - 3 } & 1.40 & 1.42 & 1.48 \\
\hline
\end{tabular}

(Viscosity of distilled water $=1.0)$

The viscosity difference due to fibrinogen maý be obtained by deducting the serum from the plasma viscosity. In order to avoid negative values for the other two fractions a deduction must first be made from the viscosity of the first and second filtrate for the contained ammonium sulphate. The viscosity of the ammonium sulphate solutions of the concentrations present in the filtrates were found to be 1.16 and 1.27 respectively. Thus the viscosity differences due to the ammonium sulphate were 0.16 and 0.27 , and the corrected viscosity of the four samples is:

\begin{tabular}{c|c|c|c}
\hline Plasma & Serum & $\begin{array}{c}\text { First Filtrate } \\
\text { less Ammonium Sulphate }\end{array}$ & $\begin{array}{c}\text { Second Filtrate } \\
\text { less Ammonium Sulphate }\end{array}$ \\
\hline 1.54 & 1.40 & 1.26 & 1.21 \\
\hline
\end{tabular}

The fibrinogen viscosity difference therefore $=1.54-1.40=0.14$; the globulin I viscosity difference $=1.40-1.26=0.14$; the globulin II viscosity difference $=1.26$ $-1.21=0.05$; and the albumin viscosity difference $=1.48-1.27=0.21$. For convenience the fractional viscosity difference will throughout the remainder of this paper be expressed as viscosity of water $=100$, so that the results of the above fractionation would be fibrinogen, 14 ; globulin I, 14 ; globulin II, 5 ; albumin, 21 . The total plasma viscosity will also be expressed as a viscosity difference, the viscosity of the contained water being deducted, giving, in my example, a figure of 54 for plasma. This will be found to equal the sum of the fractional viscosity differences.

Experimental Error.-The error involved in the actual viscosity measurement is very small and on repeated estimation does not exceed $\pm 0.4 \%$. The procedures of clotting and filtration used in the differential viscosity technique introduce additional sources of error, but the discrepancy between duplicate estimations is only slightly greater than from the simple viscosity measurement. In eight sets of duplicate 
estimations in which each sample of plasma was divided into two, and the precipitations and filtrations carried out on each separately, the maximum error in the viscosity of the filtrates was $\pm 1.0 \%$. The error which may be found when the viscosity differences are calculated is of course greater, involving as it does the combined effect of subtracting two readings to obtain a relatively small difference. In the eight sets of duplicate estimations studied the maximum errors were as follows:

\begin{tabular}{c|c|c|c}
\hline Fibrinogen & Globulin I & Globulin II & Albumin \\
\hline $\pm 2 \%$ & $\pm 5 \%$ & $\pm 25 \%$ & $\pm 6 \%$ \\
\hline
\end{tabular}

Small changes (up to 3 units) in the viscosity differences in serial estimations cannot therefore be considered significant.

As with all studies concerned with plasma proteins gross error may result from stasis induced during the collection of blood samples. For example, a sample collected during constriction of the arm at a pressure of $100 \mathrm{~mm}$. of mercury may have a viscosity $8 \%$ higher than a sample obtained at a pressure of $50 \mathrm{~mm}$., and stasis continued for one minute may result in a further increase of $8 \%$ in the viscosity. In this way the fibrinogen viscosity difference may be caused to vary by as much as $36 \%$. It is recommended that a pressure of $50 \mathrm{~mm}$. of mercury should not be exceeded in the cuff used to induce stasis and that blood be collected as soon as the cuff has been applied. The viscosity should preferably be estimated the same day, but no significant error results from leaving the plasma to stand till the following day. Delay till the third day may result in a $2 \%$ reduction of the viscosity and should therefore be avoided.

\section{Effect of Ammonium Sulphate on Viscosity of Protein Solutions}

There can be little doubt that the effect of ammonium sulphate on the viscosity of protein solutions is very much more complex than would be inferred from the simple method of calculation used. Chick (1914) found that addition of salt up to a concentration of $1 \%$ had the effect of reducing the viscosity of serum proteins. Above this concentration the addition of further salt was without effect. This reduction of viscosity, which may be termed the initial salting factor, has been determined on a number of normal and pathological samples of serum, and has been found to amount to a viscosity difference of 1 to 4 in normal samples with a range up to 9 in pathological samples. In practice, however, it was not found advantageous to include an additional stage in the fractional analysis to allow for this, as it gives little information of value.

It should be understood that no attempt has been made to estimate the actual viscosity of the fractions isolated. Normal values for the viscosity differences will be given below and these will be used for comparison with the values obtained in disease. The actual values for the viscosity of the fractions do in fact correspond with that of a pure protein solution in the case of the albumin fraction (Fig. 2), but the fibrinogen and first globulin viscosity differences are greater than the viscosity of corresponding amounts of pure fibrinogen or $\gamma$ globulin. It is unlikely that this is due in the case of globulin $I$ to the presence of small amounts of $\beta$ and $\alpha$ globulin 
in the precipitate, as the specific viscosity of globulin II is less than that of globulin I (as measured by comparing the viscosity difference and the weight of the precipitate). The effect of the ammonium sulphate accounts for a part of this discrepancy but by no means the whole. A possible explanation of the remainder may lie in the theory postulated by Harkness and Whittington (1947) of a combined protein molecule of globulin and albumin having a viscosity coefficient greater than either. The viscosity differences could, if desired, be converted into actual viscosity values of corresponding solutions of the pure protein by reference to the data in Fig. 2, but such a conversion would be of purely academic interest and of no value for routine c linical purposes. Fig. 2 could also be used to convert viscosity differences into protein concentrations of fibrinogen, $\gamma$ globu-

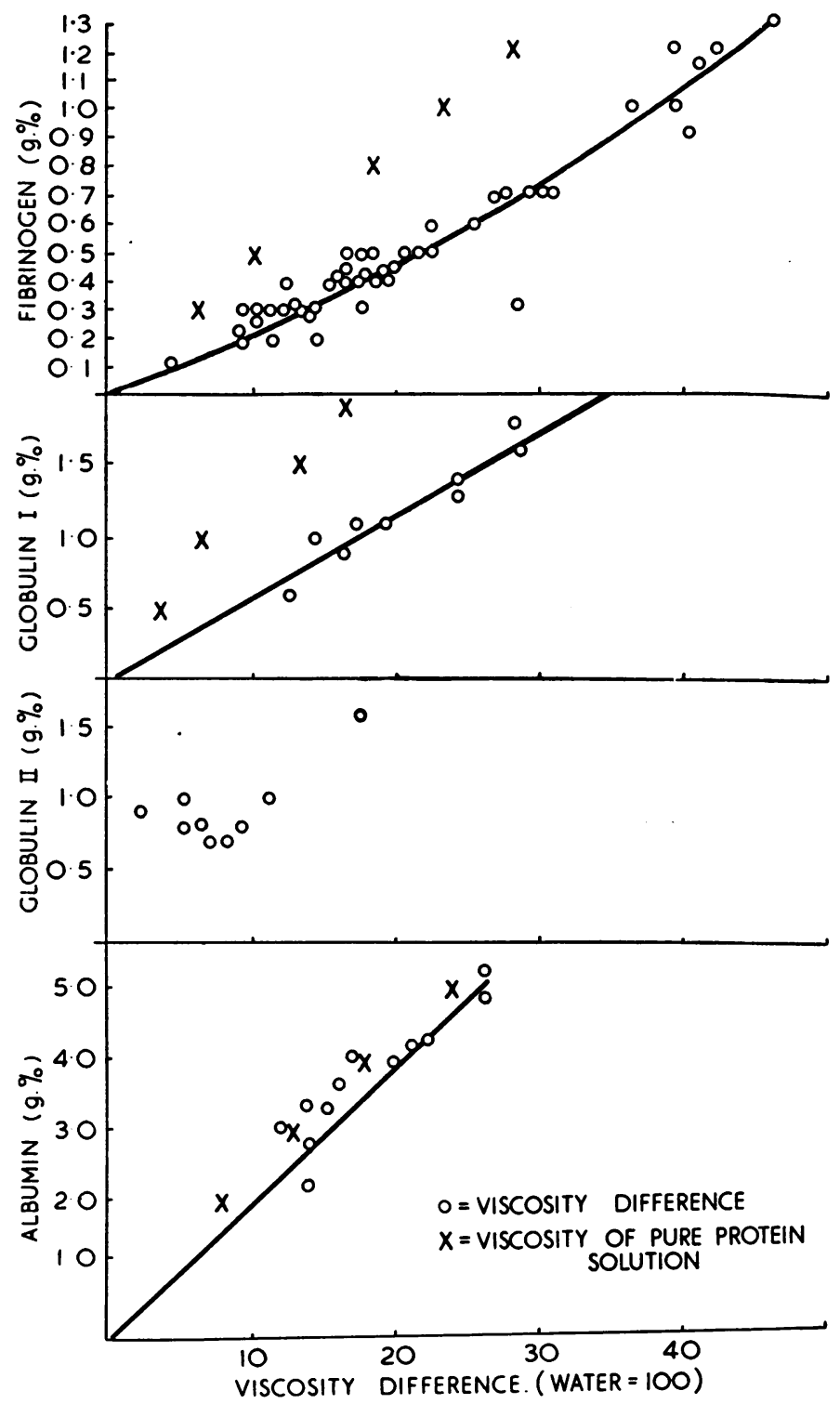

FIG. 2

lin, and albumin. As the second globulin fraction contains $a, \beta$, and $\gamma$ globulins in varying proportions no relationship between viscosity and concentration is to be expected here. The "albumin" fraction (which also contains a certain amount of a globulin) shows a closer relationship than globulin II but is less accurate than fibrinogen or globulin $I$ in the lower values. It is, however, preferable to retain the viscosity differences as such and not to attempt a conversion. The chief value 
of the method is to show relative changes and not absolute values, and these have in practice proved of great value in the detection and assessment of disease processes.

\section{Results}

Normal values were studied in 57 apparently healthy persons (mostly blood donors). Of these 36 were males and 21 females. No significant differences were found between the sexes, nor did age affect the values. The range of "normal" values encountered was as follows:

\begin{tabular}{|c|c|c|c|c|c|c|}
\hline & & Plasma & Fibrinogen & Globulin I & Globulin II & Albumin \\
\hline $\begin{array}{l}\text { Mean } \\
\text { Maximum } . . \\
\text { Minimum } \\
\text { Standard deviation }\end{array}$ & $\begin{array}{l}. . \\
\cdots \\
\cdots\end{array}$ & $\begin{array}{l}58 \\
71 \\
44 \\
10\end{array}$ & $\begin{array}{r}13 \\
19 \\
5 \\
2\end{array}$ & $\begin{array}{r}18 \\
26 \\
12 \\
6\end{array}$ & $\begin{array}{r}6 \\
10 \\
2 \\
2\end{array}$ & $\begin{array}{r}21 \\
27 \\
17 \\
6\end{array}$ \\
\hline
\end{tabular}

A brief summary of the results to be expected in disease has already been given elsewhere (Lawrence, 1949). It was pointed out that changes in the viscosity fractions were largely non-specific, being present in all processes involving tissue damage whether inflammatory, toxic, ischaemic, or due.to trauma or other physical agents, and that the relative changes in the fractions depended more on the stage of the disease process than on its nature. Thus in acute disorders the fibrinogen and sometimes the second globulin fraction were raised during the first week, the first globulin fraction being unaffected till the second or third week. During recovery the changes were found to subside in the same order, so that the first globulin fraction often remained raised for several weeks after the disease process had ceased to be active. The albumin fraction was often reduced during the early stages and returned to normal after a variable time. In subacute disorders the sequence was identical but changes took place more slowly, and in chronic disorders the fractions might remain constantly abnormal or show repeated cycles of the above sequence. These changes are illustrated by serial studies in a normal person in whom artificial fever was induced, and also in acute, subacute, and chronic disorders (Table II). The E.S.R. is also shown in order to demonstrate its failure in certain instances to reflect the changes in the plasma proteins and to indicate the presence of tissue damage. The changes following haemorrhage are also shown. Here the fractions are all reduced and the subsequent order of recovery is fibrinogen (during the first 24 hours), globulin II, albumin, and globulin I (after two weeks).

- In only a few instances could the fractional viscosity changes be said to be in any way specific. These are shown in Table III. They have already been discussed in the previous paper (Lawrence, 1949).

\section{Discussion}

The chief value of the differential plasma viscosity is that it is a comparatively simple method of indicating changes in the plasma proteins, particularly the fibrinogen and the $\gamma$ globulin. Though these changes are largely non-specific a knowledge of their presence is often of great value both in diagnosis and in assessing 
TABLE II

Serial Studies of the Differential Plasma Viscosity

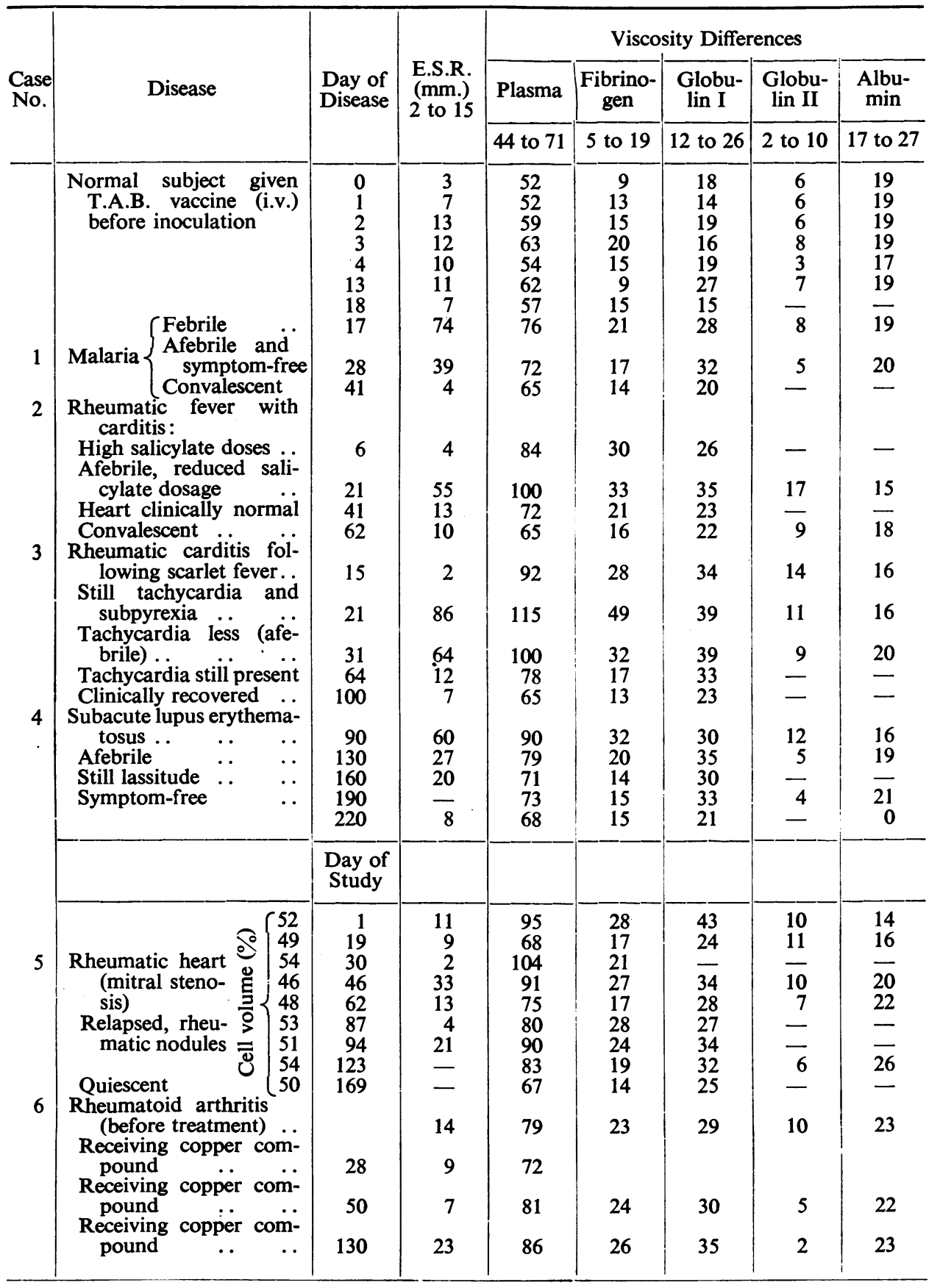


TABLE II-Continued

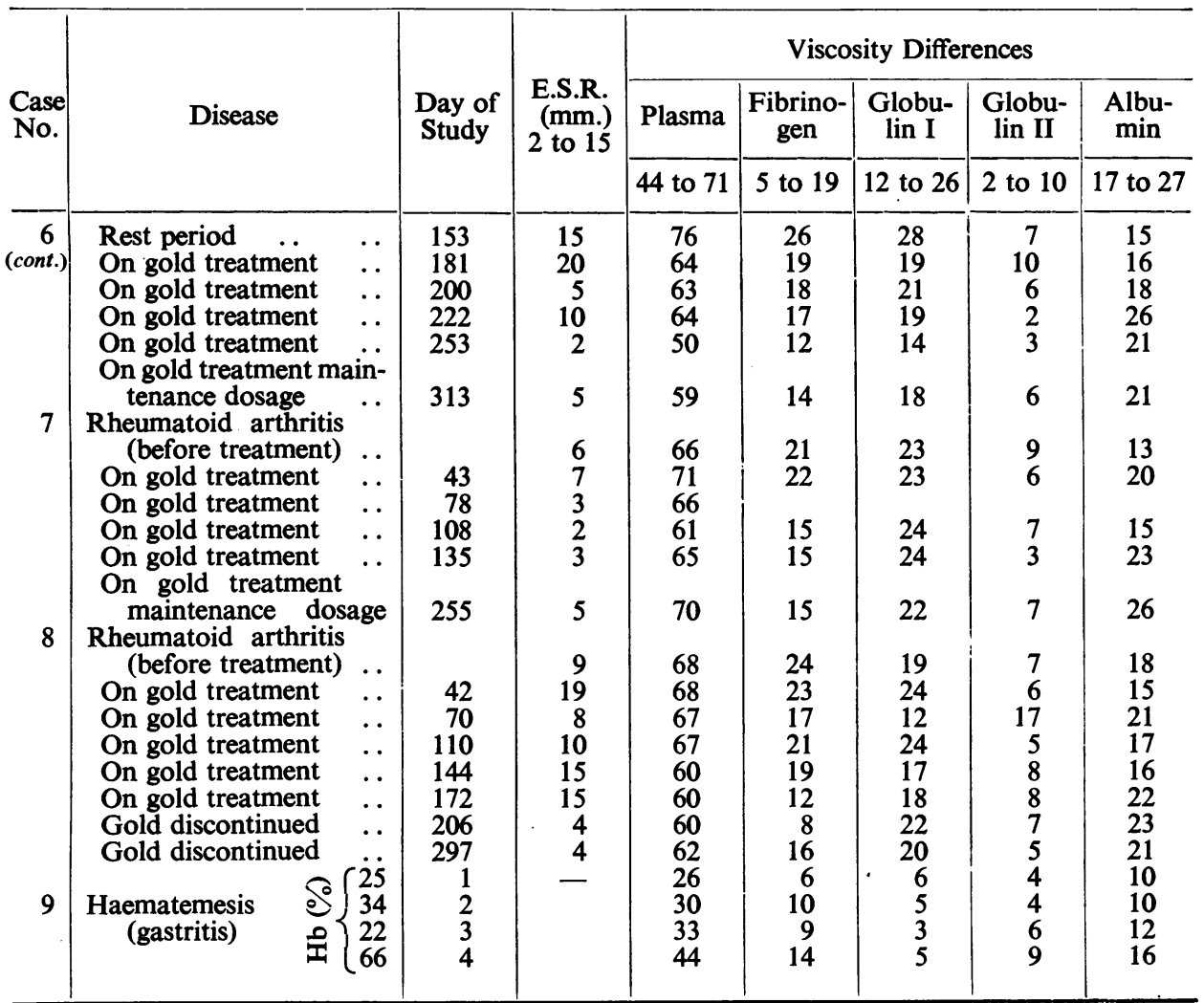

the results of therapy. Previously the E.S.R. has been largely used for this purpose, and recently the simple plasma viscosity, but each of these methods may give normal values in the presence of gross tissue damage and cannot therefore be relied on. Apart from disease of the brain, spinal cord, and meninges, which does not modify the plasma proteins, normal values for the differential plasma viscosity may occasionally be found in localized disease such as appendicitis, furuncle, or small malignant tumours if without metastases, but is never normal when widespread active disease is present. Space does not permit of a full discussion of this aspect, but an indication of the relative value of the E.S.R. and the differential and total plasma viscosity will be found in the previous paper.

The test is also of value in the diagnosis and assessment of blood loss, particularly where this has been occult as in gastric and duodenal haemorrhage. The rapid return of the fibrinogen viscosity level to normal when haemorrhage has ceased gives a ready indication of progress, and the slow recovery of the first globulin fraction gives useful confirmation of a belated diagnosis of occult haemorrhage.

In addition, specific changes indicated in Table III, the very high globulin I and fibrinogen in myelomatosis, the high globulin $I$ in liver disease, and the low 
TABLE III

Possible Diagnostic Value of Certain Differential Plasma Viscosities

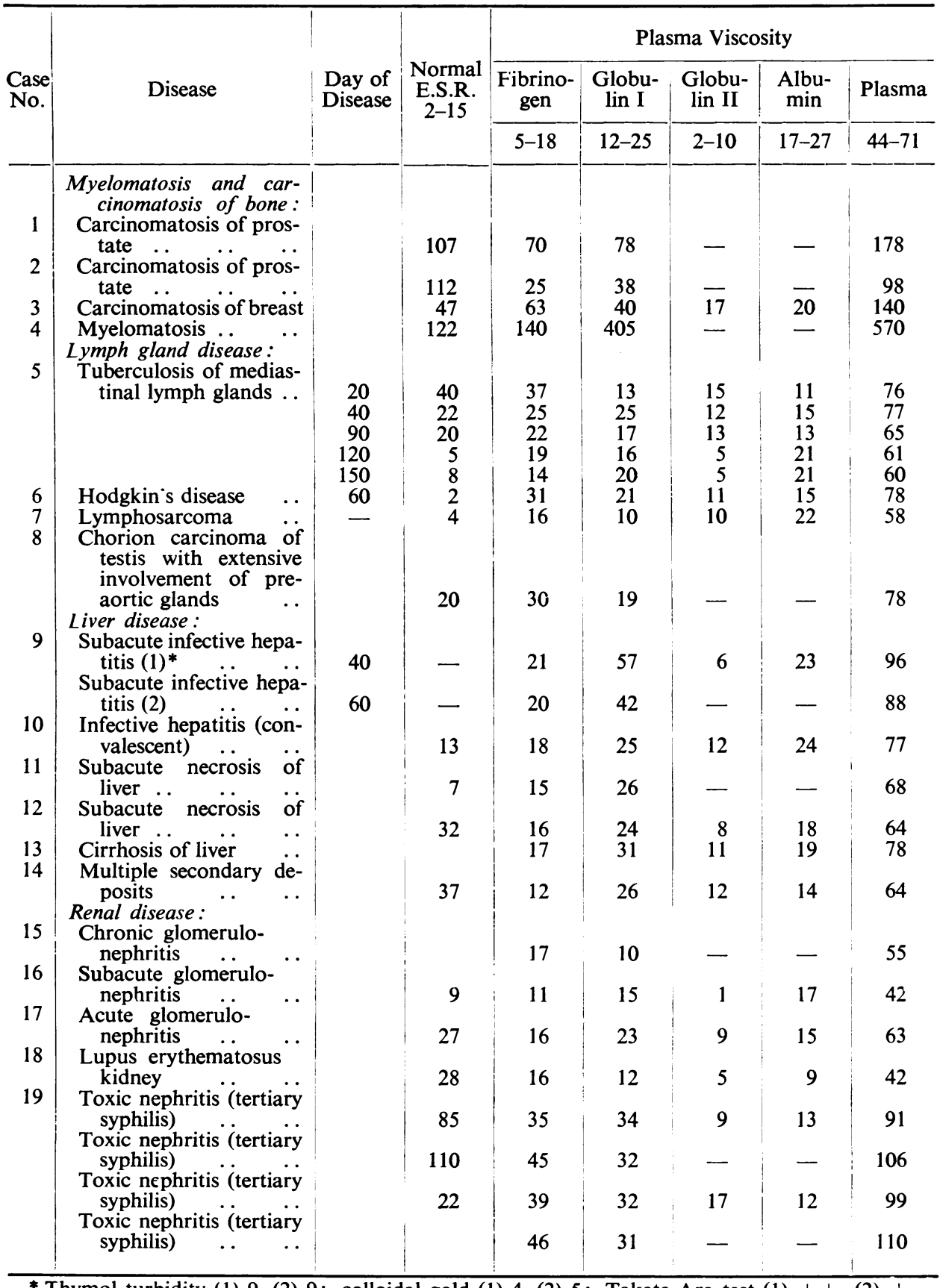

\footnotetext{
* Thymol turbidity (1) 9, (2) 9; colloidal gold (1) 4, (2) 5; Takata-Ara test (1),$++(2)+$.
} 
value or lack of increase of this fraction in lymph gland disease may occasionally assist in diagnosis, but the information which the test gives as to the stage of disease is more often of value.

\section{Summary}

Technical aspects of the measurement of the plasma viscosity are discussed with particular reference to suitable types of instrument and their calibration for use with protein solutions.

A method for estimating the viscosity of individual protein fractions is described, and values found in 47 healthy individuals are noted.

From a study of 700 samples in a wide variety of diseases it is concluded that the differential plasma viscosity gives useful information as to changes in the plasma proteins, particularly the fibrinogen and $\gamma$ globulin fractions, and that it is a more reliable indicator of these changes than the E.S.R. or the simple plasma viscosity. The changes in the differential viscosity at different stages of disease are noted and their non-specific nature stressed.

Possible diagnostic applications are suggested.

I wish to thank Professor R. B. Whittington of the University of Minnesota for his kind assistance and guidance in the intricate problems of viscometry, and Dr. J. H. Kellgren, Director of the Rheumatism Research Centre, Manchester University, and Professor S. L. Baker for their interest and advice.

\section{REFERENCES}

Chick, H. (1914). Biochem. J., 8, 261.

Fåhraeus, R. (1921). Acta med. scand., 55, 1.

Harkness, J., and Whittington, R. B. (1947). Biochim. biophys. Acta, 1, 487.

Houston, J., Harkness, J., and Whittington, R. B. (1946). Acta tuberc. scand., 19, 153.

Jager, B. V., and Nickerson, M. (1948). J. clin. Invest., 27, 231.

Lawrence, J. S. (1949). Ann. rheum. Dis., 8, 209.

Miller, A. K., and Whittington, R. B. (1942). Lancet, 2, 510.

T'Ang, B. H. Y., and Wang, S. H. (1940). Chin. med. J., 57, 546. 